and safe coverage of the microvascular anastomoses, including direct skin closure, skin grafting and flap coverage..$^{[1,2]}$ In our experience, pointing out indications and surgical tips, split-thickness skin graft (STSG) is a safe method for permanent and/or transient vascular pedicle coverage. It can be used to avoid three compromising situations, namely pedicle compression, pedicle exposure and pedicle infection and hence we support the idea of covering the pedicle with split skin graft.

1. Coverage of elective free flaps vascular pedicle to avoid tension closure: Our experience suggests that a skin graft is safer than direct closure with moderate tension, especially in lower limb and overweight patients. STSG must be very thin, can be left until final healing [Figure 1] or replaced if hematoma occurs underneth or excised after oedema resolution to allow direct closure; scar revision improves the aesthetic outcome.

2. Coverage of exposed vessels and nerves in replantation and complex revascularization: When these vital structures are exposed due to soft tissue debridement, a thin STSG can be used to temporarily cover them; the graft is removed and changed during next debridements until definitive flap coverage in the next few days. This undoubtedly is the best biological dressing.

\title{
Microvascular pedicle coverage with split thickness skin graft: Indications and surgical tips
}

Sir,

Free flap failure is a traumatic event for both the patients and the surgeon and complications can be reduced to a minimum by ensuring correct planning and execution of surgical technique and better postoperative care. In free tissue transfer, the surgeon must consider and avoid the various factors that can reduce blood flow across the anastomoses after microsurgical repair. Prevention of kinking of the vascular tension-free pedicle is imperative but not enough because inadequate and compressive soft tissue closure and scant protection around the microvascular anastomosis predispose to thrombosis and flap failure. ${ }^{[1-3]}$ In addition, skin is often missing or insufficiently lax to allow primary closure or subcutaneous tunneling of the pedicle in complex tissue defects. ${ }^{[3]}$

Different techniques are described to obtain satisfactory
3. Coverage of vessels in deep wound sepsis: After radical debridement of all non viable tissues it is vital to protect the vascular and neural repairs or intact nerves nad vessels. Thin split thickness skin graft are used temporarily to cover them; the graft is removed and changed daily or every two days until infection resolves. A free flap can then be planned to close the wounds.

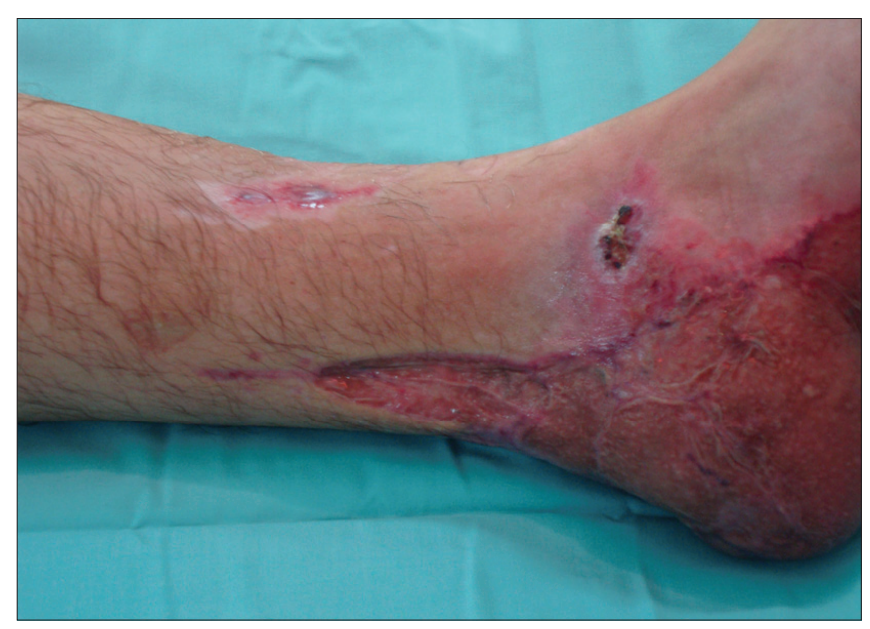

Figure 1: STSG healed on a free flap vascular pedicle used for an inferior limb reconstruction 
4. Transient coverage of vein grafts or staged vascular loops. In case of STSG covering vein graft to artery, it is advisable to provide a more stable coverage as soon as possible to avoid bleeding from minor traumas. ${ }^{[4]}$

Proper microsurgical skills minimize complications but even a perfect anastomosis will fail if the pedicle is not well positioned and protected from compression, hematoma, kinking, tunneling or skin deficiency, all of which increase the risk of vessel thrombosis. To avoid these dynamic and positional complications, temporary or permanent STSG of the pedicle should be considered. After insetting a flap, a limb revascularization or replantation, the following mental check list for vessel positioning is useful: A tension free pedicle with normal forward flow, no redundancy (especially for veins), no compressions, no kinks, no twists and viable non-infected tissues around vascular or nerves repairs.

Although complications in free tissue transfer surgery cannot be completely prevented, STSG can aid in avoiding complications related to poor coverage of the pedicle.

Thione A., Cavadas P. C., Landin L., Ibañez J. Clinica Cavadas, Plastic and Reconstructive Surgery, Valencia, Spain

Address for correspondence: Dr. Alessandro Thione, Plastic and Reconstructive Surgery, Clinica Cavadas, Paseo de Facultades 1, bajo 8, 46021 Valencia, Spain. E-mail: althione@email.it

\section{REFERENCES}

1. Ohtsuka H, Hwang HY, Torigai K, Shioya N. Methods of vascular pedicle coverage. Ann Plast Surg 1979;3:315-20.

2. Riboh J, Nigriny J, Chong A, Page R, Chang J. Optimization of microsurgery: Improved coverage of the latissimus dorsi vascular pedicle with vascularized serratus fascia. Ann Plast Surg 2007;58:109-11.

3. Cavadas PC. Salvage of replanted upper extremities with major soft tissue complications. J Plast Reconstr Aesthet Surg 2007;60:769-75.

4. CavadasPC.Arteriovenousvascularloopsinfreeflap reconstruction of the extremities. Plast Reconstr Surg 2008;121:514-20.

\begin{tabular}{|l|l|}
\hline \multicolumn{3}{|c|}{ Access this article online } \\
\hline Quick Response Code: & Website: \\
\hline & www.ijps.org \\
\hline & \\
\hline
\end{tabular}

